SUBJECT AREAS:

ASTROPHYSICAL

PLASMAS

STRUCTURE OF SOLIDS AND

LIQUIDS

CORE PROCESSES

Received

16 April 2014

Accepted

11 June 2014

Published

27 June 2014

Correspondence and requests for materials should be addressed to J.D. (jydai@nudt.edu. cn) or J.Y. (jmyuan@ nudt.edu.cn)

\section{Nuclear quantum dynamics in dense hydrogen}

\author{
Dongdong Kang' ', Huayang Sun', Jiayu Dai' ', Wenbo Chen' ', Zengxiu Zhao', Yong Hou', Jiaolong Zeng' \\ \& Jianmin Yuan ${ }^{1,2}$
}

\begin{abstract}
'Department of Physics, College of Science, National University of Defense Technology, Changsha 410073, Hunan, People's Republic of China, ${ }^{2}$ State Key Laboratory of High Performance Computing, National University of Defense Technology, Changsha 410073, Hunan, People's Republic of China.
\end{abstract}

Nuclear dynamics in dense hydrogen, which is determined by the key physics of large-angle scattering or many-body collisions between particles, is crucial for the dynamics of planet's evolution and hydrodynamical processes in inertial confinement confusion. Here, using improved ab initio path-integral molecular dynamics simulations, we investigated the nuclear quantum dynamics regarding transport behaviors of dense hydrogen up to the temperatures of $1 \mathrm{eV}$. With the inclusion of nuclear quantum effects (NQEs), the ionic diffusions are largely higher than the classical treatment by the magnitude from $20 \%$ to $146 \%$ as the temperature is decreased from $1 \mathrm{eV}$ to $0.3 \mathrm{eV}$ at $10 \mathrm{~g} / \mathrm{cm}^{3}$, meanwhile, electrical and thermal conductivities are significantly lowered. In particular, the ionic diffusion is found much larger than that without NQEs even when both the ionic distributions are the same at $1 \mathrm{eV}$. The significant quantum delocalization of ions introduces remarkably different scattering cross section between protons compared with classical particle treatments, which explains the large difference of transport properties induced by NQEs. The Stokes-Einstein relation, Wiedemann-Franz law, and isotope effects are re-examined, showing different behaviors in nuclear quantum dynamics.

$\mathrm{N}$ uclear quantum nature, especially for protons, manifests its significant effects on the ionic structures, phase diagram and ionic dynamics ${ }^{1-5}$. A lot of researches focus on the nuclear quantum effects (NQEs) such as quantum fluctuations and zero-point motion on the structures of dense hydrogen ${ }^{6-10}$, revealing many intriguing physics. However, the nuclear quantum dynamics such as ionic quantum transport is rarely studied because of the computational and the theoretical challenges. Only at low temperatures, the nuclear quantum dynamics was investigated. For example, large deviation for ionic diffusion and thermal conductivity of para-hydrogen is found below $32 \mathrm{~K}$ induced by NQEs compared with the classical-particle treatment ${ }^{11}$. At high temperatures, in particular in warm dense regime (i.e., above $1000 \mathrm{~K}$ ), ions are usually considered as distinguishable classical particles by assuming the quantum fluctuations or quantum collisions are negligible. However, even for the collision energies up to $10 \mathrm{eV}$, it has been shown that elastic collision cross sections for protons and hydrogen atoms can be very different in the quantum and classical theory ${ }^{12}$, indicating the possible failure of classical treatment for nuclear motion in warm dense regime.

Dense hydrogen plays a crucial role in understanding the material behaviors under extreme conditions ${ }^{13,14}$ and has important applications in energy sources ${ }^{15}$ and astrophysics ${ }^{16,17}$. The associated ionic and electronic transport properties are the key to tracing the dynamics of capsule implosion of inertial confinement fusion (ICF) ${ }^{15}$, modeling plasma processes ${ }^{18}$ and the interior structure and evolution of giant planets and exoplanets ${ }^{19-21}$. More exact transport properties such as thermal conductivities and electron-ion relaxation rates are required because they can remarkably alter the dynamics of planet's evolution ${ }^{22}$ and hydrodynamical processes in ICF $^{23}$. The core pressures of some newly discovered exoplanets are estimated even up to $19 \mathrm{Gbar}^{21}$, while the temperature may be several thousand Kelvins. Thanks to the recent progress in experimental techniques, one could get access to this previously inaccessible regime of phase diagram of hydrogen through a laser-induced shock wave loading of precompressed samples ${ }^{24,25}$. However, theoretically understanding the nature of hydrogen under the ultra-high pressures is still rare and a great challenge. Density functional theory based ab initio molecular dynamics (MD) has been widely used to investigate transport properties such as ionic diffusion, electrical conductivity of dense matter at high temperatures ${ }^{26-28}$. In viewpoint of collision physics, both the ionic and electronic transport properties depend strongly on the scattering cross sections between particles. In usual $a b$ initio MD simulations, the corresponding scattering cross sections are statistically obtained from classical particle 
dynamics simulations, in which the quantum dynamics such as tunnelling is absent. The role of quantum dynamics in dense hydrogen is of great interest and expected to give a new perspective on NQEs.

Path-integral molecular dynamics (PIMD) with imaginary-time path discretization has provided a proper theoretical description of the nuclear quantum fluctuation and delocalization ${ }^{29,30}$. Further, the adiabatic centroid PIMD is proposed to obtain the real-time dynamics of nuclei in the quasiclassical approximation ${ }^{31}$. Its accuracy has been extensively studied for the bound systems $\mathrm{s}^{32,33}$. However, for the continuum states, for example quantum tunnelling through a potential barrier, whether the centroid PIMD is accurate is already an open question. Since the ionic dynamics is strongly dependent on the processes of both bound and continuum states, it is urgent to understand this issue, looking forward to reach progress.

In this work, we further developed the adiabatic centroid PIMD approach and the accuracy was demonstrated from quantum tunnelling of a particle through the potential barrier. Within this theoretical framework, the nuclear quantum dynamics of dense hydrogen was investigated, showing more interesting intrinsic physics. Both the ionic and electronic transport properties were greatly affected by NQEs, which enables us to understand the properties of dense matter more profoundly.
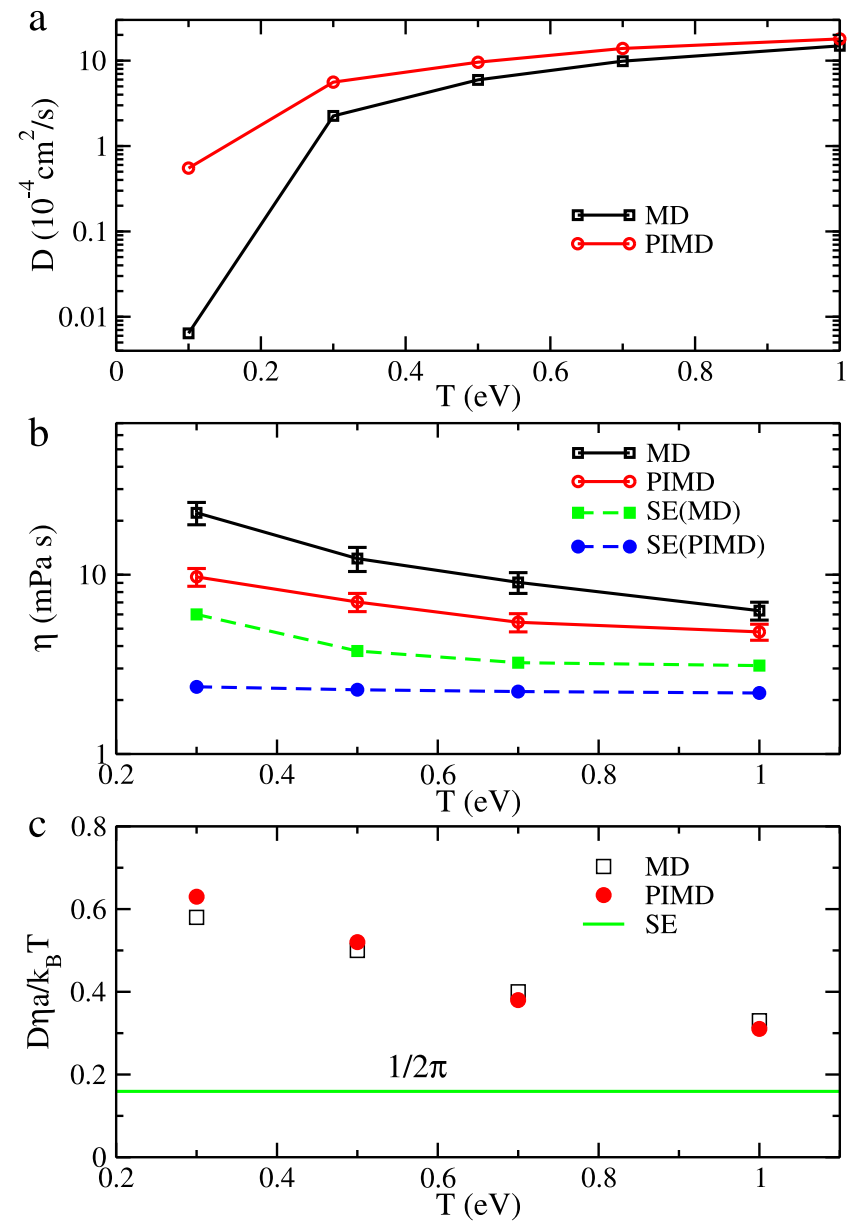

Figure $1 \mid$ Temperature dependence of the self-diffusion coefficient (a) and shear viscosity (b) at $10 \mathrm{~g} / \mathrm{cm}^{3}$ obtained from centroid PIMD and MD simulations. The viscosities obtained from the Stokes-Einstein (SE) relation are also presented. (c) Examination of SE relation with respect to temperature. The effective atomic diameter in SE relation is defined by the position of the first peak of radial distribution function.

\section{Results}

Structure and ionic transport properties. For dense hydrogen, five state points along the $10 \mathrm{~g} / \mathrm{cm}^{3}$ isochore from $0.1 \mathrm{eV}$ to $1 \mathrm{eV}$, and three state points along the $1 \mathrm{eV}$ isotherm from $10 \mathrm{~g} / \mathrm{cm}^{3}$ to $100 \mathrm{~g} /$ $\mathrm{cm}^{3}$ were studied. The main motivation for choice of these parameters is that in ICF a solid cryogenic shell of DT fuel in capsule needs to pass through a low temperature and high density stage before achieving the final state of the high density and temperature $^{34}$. The transport properties at this stage are essential to understand the whole implosion process. Besides, the cores of some exoplanets are likely to be under the conditions studied here ${ }^{13}$. The structural properties were calculated using the primitive scheme ${ }^{29}$ of PIMD, and the real-time quantum dynamics of nuclei was obtained through the improved $a b$ initio centroid PIMD (see Methods). Exchange effects between protons are neglected in this study ${ }^{35}$. Firstly, we calculated the self-diffusion coefficient and the shear viscosity at $10 \mathrm{~g} / \mathrm{cm}^{3}$ with temperatures from $0.1 \mathrm{eV}$ to $1 \mathrm{eV}$ and the results are shown in Fig. 1a and $1 \mathrm{~b}$. When taking into account the quantum collision properly, the ionic diffusion is much higher than that with classical treatment for ions. We can see that the selfdiffusion coefficient at $0.1 \mathrm{eV}$ from the centroid PIMD simulations is two orders of magnitude larger than the MD value. This large increment is likely to arise from the phase transition from solid to liquid with the inclusion of NQEs, which is confirmed directly by ionic trajectory analyses (see the Supplementary Information for simulated movies). The difference introduced by NQEs is also clearly demonstrated by radial distribution functions (RDFs) of hydrogen nuclei in Fig. 2a. Note that the RDF of hydrogen nuclei with MD method exhibits long-range ordered solid-like character at the low temperature of $0.1 \mathrm{eV}$, which is not visible in RDF obtained from the PIMD calculations. In the quantum-mechanical perspective, it can be understood from that the protons tunnel through the energy barriers and move away from their equilibrium positions in crystal structure at $0.1 \mathrm{eV}$, as appeared in hydrogenbonded systems at low temperatures ${ }^{5}$. Our results accord with the conclusions in Ref. 36, i.e., the melting temperature of hydrogen is lowered by NQEs. In addition, we note that although hydrogen is in solid state at $0.1 \mathrm{eV}$ in classical simulation, the self-diffusion coefficient of protons does not vanish, which arises from the
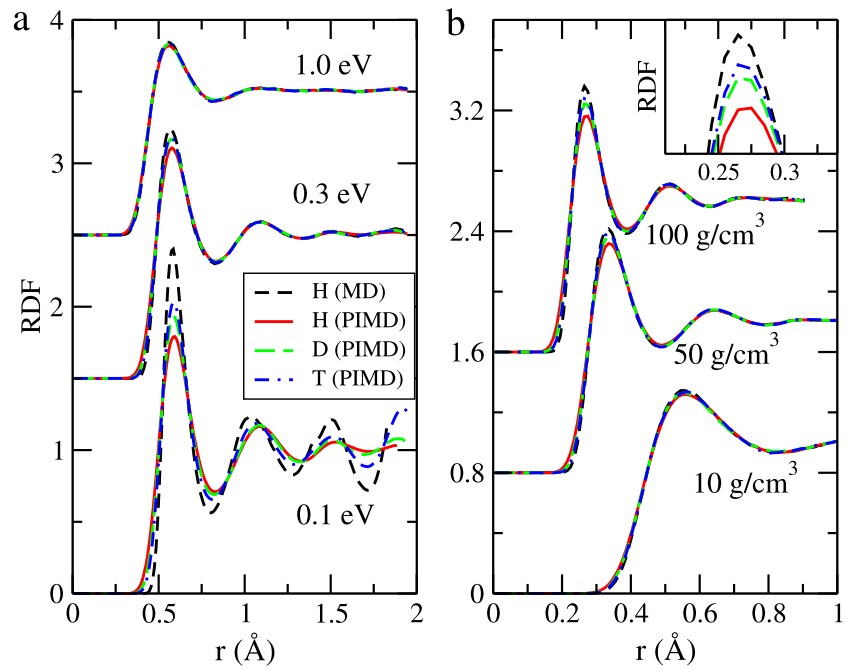

Figure $2 \mid$ Radial distribution functions of hydrogen nuclei from PIMD (solid lines) and MD (dashed lines) simulations with different temperatures at $10 \mathrm{~g} / \mathrm{cm}^{3}$ (a), and with different densities at $1 \mathrm{eV} \mathrm{(b).} \mathrm{The}$ isotopes effects of deuterium (D) (long-dashed lines) and tritium (T) (dotdashed lines) are also presented. The inset is the first peak of radial distribution functions at $100 \mathrm{~g} / \mathrm{cm}^{3}$ and $1 \mathrm{eV}$. 
presence of atomic diffusion in solid hydrogen when the temperature is close to melting point ${ }^{37}$.

The self-diffusion coefficients obtained via centroid PIMD are substantially larger than the classical particle value over the whole temperature range of $0.1-1 \mathrm{eV}$, as shown in Fig. 1a. The difference is from $146 \%$ at $0.3 \mathrm{eV}, 61 \%$ at $0.5 \mathrm{eV}$ to $40 \%$ at $0.7 \mathrm{eV}$. There is still a large difference of $20 \%$ even at the high temperature of $1 \mathrm{eV}$. It is not open-and-shut because the RDFs from the MD and PIMD calculations are very close at $0.3 \mathrm{eV}$ and almost identical when the temperature is up to $1 \mathrm{eV}$ (see Fig. 2a). We note that the large-angle scattering between ions is dominant at such high densities considered here. The pronounced quantum nuclear wave scattering calculation leads to a smaller large-angle scattering cross section between ions than the classical particle scattering calculation, and thus increases the mean free path of ions. Therefore, the ions diffuse more easily and the classical particle treatment of protons substantially underestimates the ionic diffusion.

The shear viscosities $\eta$ calculated from the Green-Kubo relation ${ }^{27}$ at the density of $10 \mathrm{~g} / \mathrm{cm}^{3}$ are shown in Fig. 1b. Corresponding to the diffusions $D$, the viscosities from centroid PIMD are smaller than those from $\mathrm{MD}$, but they become closer at high temperatures. In addition, the viscosities are also deduced from the Stokes-Einstein (SE) relation with slip boundary condition ${ }^{38}$. As discussed in Ref. 39, the SE relation, which is exact for the Brownian particles, is not valid in strongly coupled regime. It is shown in Fig. 1c that the values of $D \eta a / k_{B} T$ ( $a$ is the effective atomic diameter and defined by the position of the first peak of RDF) from both MD and PIMD calculations are much higher than the SE relation value $1 / 2 \pi$. At low temperatures, pronounced NQEs induce more deviation from SE relation, indicating that the correlated time of particles in PIMD simulations (non-Markov dynamics) is longer than that of classical treatment.

The largely reduced and broadened first peak of RDF from PIMD calculations at $0.1 \mathrm{eV}$ and $10 \mathrm{~g} / \mathrm{cm}^{3}$ indicates the significant nuclear quantum delocalization, which becomes weaker with increasing temperature and cannot be observed at $1 \mathrm{eV}$ finally. Besides, RDFs show much pronounced nuclear quantum character with increasing density (see Fig. 2b). When the density is increased up to $100 \mathrm{~g} / \mathrm{cm}^{3}$, there is a distinct difference of RDFs between the PIMD and MD calculations due to NQEs even at the high temperature of $1 \mathrm{eV}$. Meanwhile, the isotope substitutions provide a clear demonstration of isotope effects on nuclear spacial distribution of condensed hydrogen. Since the tritium nuclei is the heaviest one and has the shortest thermal de Broglie wavelength, RDFs of the tritons are more structured than those of the other two isotopes.

Electronic transport properties. Of our particular interest is whether the NQEs have evident effects on the electronic transport properties as profound as nuclei exhibits. To shed light on this issue, we calculated the electrical conductivity and optical absorption coefficient of dense hydrogen. In order to avoid the artificial drop at low frequency due to the finite number of atoms in the simulations, we employed the Drude formula to estimate the dc conductivity at a zero frequency limit ${ }^{26}$. It can be clearly seen from Fig. 3 that with the inclusion of NQEs, the low-frequency dependent electrical conductivity $\sigma(\omega)$ exhibits a different trend compared with the results from the MD simulations, which directly leads to the different dc conductivity $\sigma_{\mathrm{dc}}(\omega \rightarrow 0)$ except for the state point of $10 \mathrm{~g} / \mathrm{cm}^{3}$ and $1 \mathrm{eV}$. The dc conductivities of dense liquid hydrogen from the PIMD calculations are largely suppressed compared with the MD value by $25 \%$ at $10 \mathrm{~g} / \mathrm{cm}^{3}, 0.3 \mathrm{eV}$ and $18 \%$ at $100 \mathrm{~g} / \mathrm{cm}^{3}$, $1 \mathrm{eV}$. A visible larger optical absorption for hydrogen of $10 \mathrm{~g} / \mathrm{cm}^{3}$ at $0.3 \mathrm{eV}$ and $100 \mathrm{~g} / \mathrm{cm}^{3}$ at $1 \mathrm{eV}$ is displayed in Fig. 3. It should be noted that the differences at $10 \mathrm{~g} / \mathrm{cm}^{3}$ and $0.1 \mathrm{eV}$ originate from the different structures (solid in classical simulation and liquid in quantum simulation mentioned above). It is well known that NQEs will introduce more disorder for the systems, lowering the

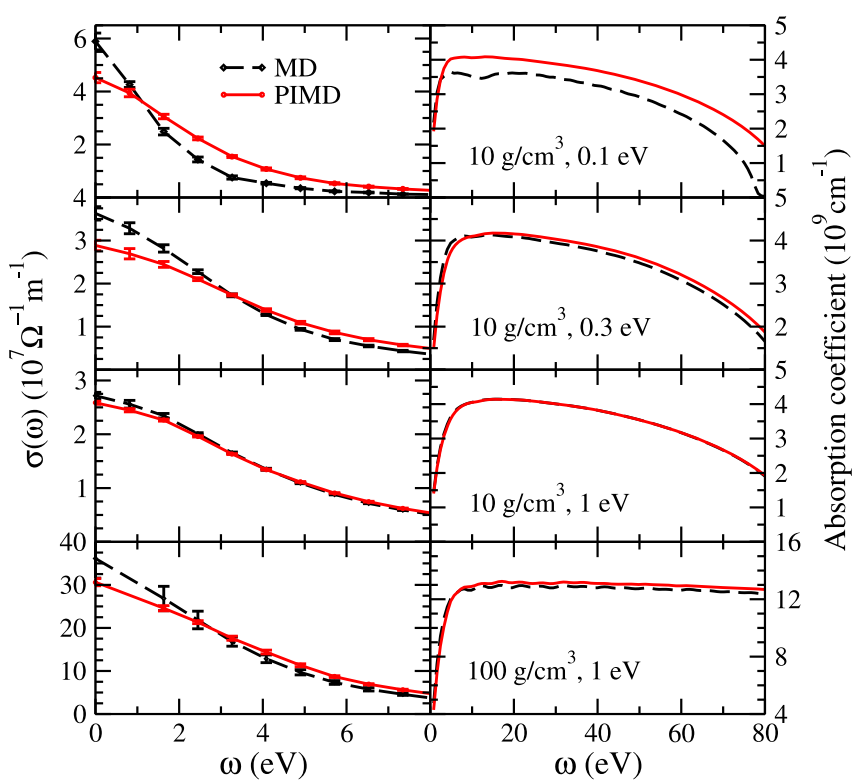

Figure 3 Electrical conductivity and optical absorption coefficient from the PIMD (solie lines) and MD (dashed lines) calculations for four state points, i.e., $\left(10 \mathrm{~g} / \mathrm{cm}^{3}, 0.1 \mathrm{eV}\right)$ (upper panel), $\left(10 \mathrm{~g} / \mathrm{cm}^{3}, 0.3 \mathrm{eV}\right)$ (second panel), $\left(10 \mathrm{~g} / \mathrm{cm}^{3}, 1 \mathrm{eV}\right)$ (third panel), $\left(100 \mathrm{~g} / \mathrm{cm}^{3}, 1 \mathrm{eV}\right)$ (lower panel). The error bars denote the standard deviation of averaging 10 atomic configurations along the molecular dynamics trajectory. Note that the structure from MD simulation at the state point of $\left(10 \mathrm{~g} / \mathrm{cm}^{3}, 0.1 \mathrm{eV}\right)$ is solid.

melting point and critical point ${ }^{9}$. In fact, NQEs introduce ionic delocalization and closer ionic distances, as shown in Fig. 2, which produce more localized electrons around the nuclei, evidenced by the distribution of charge density and electron localization function (see Fig. 4). The stronger localized electrons might enhance the optical oscillator strength and optical absorption cross sections of relative high frequencies. In addition, NQEs cause nonuniform broadening of energy bands because of the anharmonic effects, resulting significant difference in the electronic density of states (DOS). In particular, the DOS around Fermi level are decreased by NQEs (See the Supplementary Information).

In Fig. 5, it is shown that the electronic thermal conductivities with the inclusion of NQEs are smaller than the value obtained from MD simulations as the electrical conductivity exhibits. We also computed the Lorenz number which is the ratio between electrical and thermal conductivity divided by the temperature ${ }^{40}$. According to the Wiedemann-Franz law, the Lorenz number is constant and reaches $2.44 \times 10^{-8} \Omega \mathrm{WK}^{-2}$ for the degenerate and coupled plasma ${ }^{41}$. Compared to the good agreement with Wiedemann-Franz law for the MD results, the Lorenz number with the inclusion of NQEs deviates slightly from the ideal value at low temperatures, where the ionic structures are significantly different.

\section{Discussion}

We have investigated the nuclear quantum dynamics in transport behaviors of dense hydrogen using the improved centroid PIMD simulations. The complex transport behavior shows that even when the NQEs affect little on the static structures $\left(10 \mathrm{~g} / \mathrm{cm}^{3}\right.$ and $\left.1 \mathrm{eV}\right)$, the ionic diffusions are still largely higher due to the lower collision cross sections with the inclusion of NQEs. In addition, electrical and thermal conductivities have a pronounced decrease in quantum simulations as well. The SE relation is not valid in strongly coupled regime, and the Lorenz number deviates from the degenerate limit at low temperatures. We have shown that the quantum nuclear character 
a

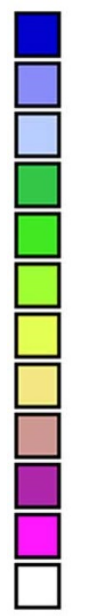

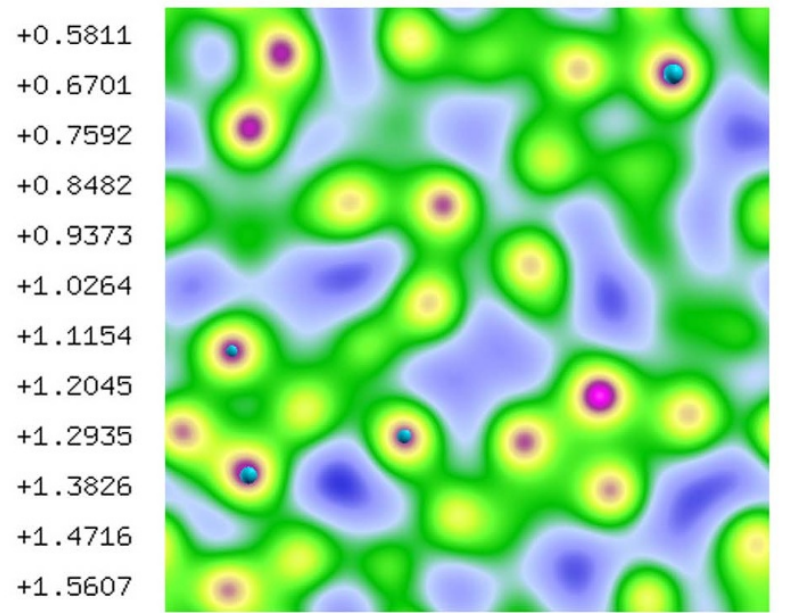

\section{C}

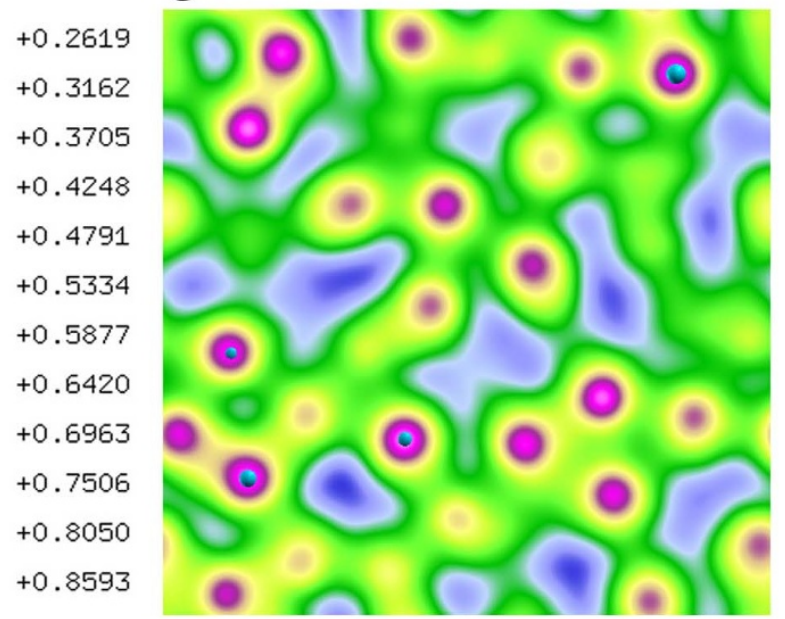

b

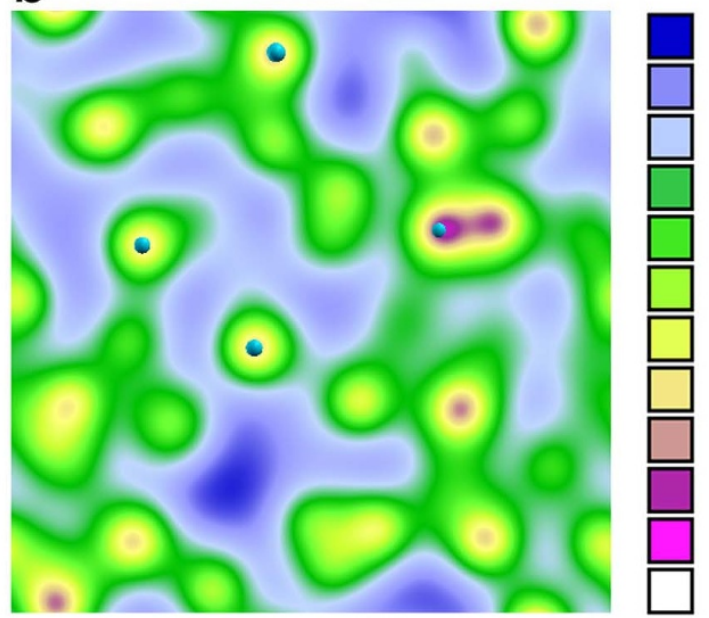

$+0.4752$

$+0.6008$

$+0.7264$

$+0.8520$

$+0.9776$

$+1.1033$

$+1.2289$

$+1.3545$

$+1.4801$

$+1.6058$

$+1.7314$

$+1.8570$

d

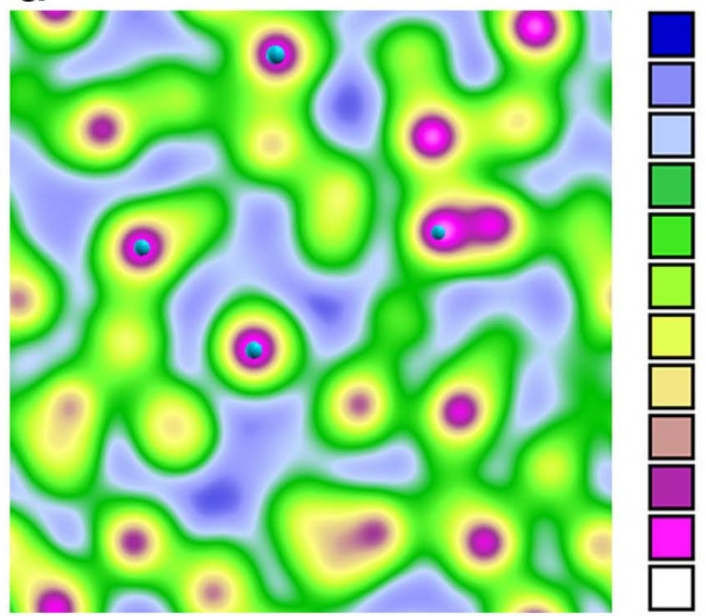

$+0.2452$

$+0.3034$

$+0.3616$

$+0.4198$

$+0.4780$

$+0.5362$

$+0.5944$

$+0.6526$

$+0.7108$

$+0.7690$

$+0.8272$

$+0.8854$

Figure 4 The cut-plane of 3D distribution of charge density $(a, b)$ and electron localization function (c, d) of randomly selected configurations from MD (a, c) and PIMD (b, d) simulations at $10 \mathrm{~g} / \mathrm{cm}^{3}$ and $0.3 \mathrm{eV}$. In PIMD calculations, the configuration is randomly selected from imaginary-time slices in PIMD time steps. Note that with the inclusion of NQEs, the charge density and electron localization function have the larger maxima and smaller minima compared to the classical simulations. It indicates that electrons surrounding ions distribute more localized with quantum nuclei than classical particle treatment. All the other randomly selected configurations exhibit the same behavior as the example presented here.

induces complex behaviors for both the ionic and electronic transport of dense hydrogen.

\section{Methods}

Centroid path-integral molecular dynamics for quantum tunneling. In the primitive PIMD formalism ${ }^{29}$, the $\mathrm{N}$-particle quantum system is isomorphic to a classical system consisting of $N$ ring polymers of length $P$ with harmonic intrapolymeric forces. Thus the static properties of the quantum system can be obtained through MD simulations of its isomorphic classical system. In the adiabatic centroid PIMD scheme ${ }^{31}$, the normal mode transformation diagonalizes the harmonic bead coupling. The centroid and non-centroid modes are mass-scale separated. The nuclear quantum dynamics is obtained in the quasiclassical approximation through driving the centroid move in real-time in the centroid effective potential generated by all non-centroid modes. Taking for example the case of one kind of particle in the system, the coefficient of stiffness of harmonic interaction is $\omega_{P}=\sqrt{P} k_{B} T=\frac{2 N}{g} \sqrt{P} E_{0}$, where $P$ is the number of beads in the ring polymer, $k_{B}$ is the Boltzmann constant, $T$ is the target temperature of the simulated system, $g$ is the total degrees of freedom of ions, $E_{0}$ is the average kinetic energy of each ion determined by the target temperature $T$. In this scheme, $\omega_{P}$ keeps constant for a given temperature $T$ regardless of the circumstance of the ions.

Here we propose a new scheme in order to make the size of ring polymer is more close to $\lambda$ as the instantaneous kinetic energy of ions is decreased. For each ion, $\omega_{P}$ depends linearly on its instantaneous kinetic energy $E(t)$ during simulations,

$\omega_{P}(t)=\frac{2 N}{g} \sqrt{P}\left(a E(t)+b E_{0}\right)$, where $t$ is the simulation time step, two parameters $a$

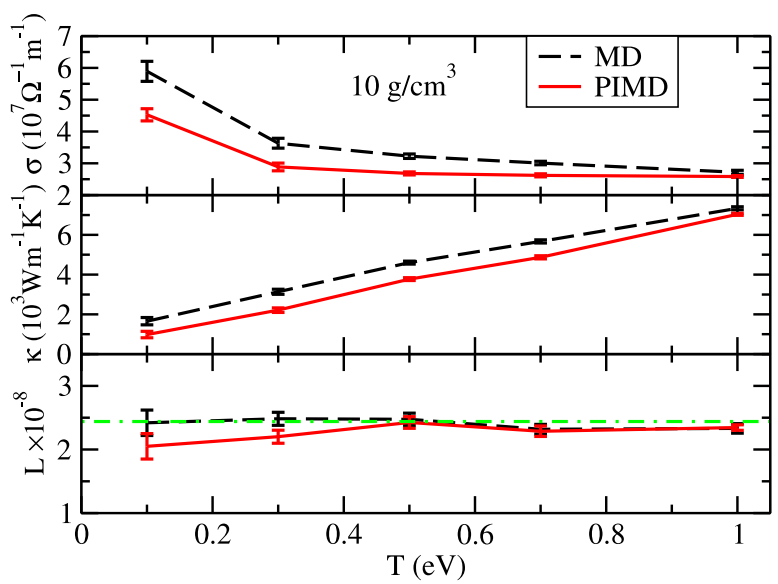

Figure 5 | Electrical conductivity, thermal conductivity and Lorenz number of dense hydrogen as a function of temperature at $10 \mathrm{~g} / \mathrm{cm}^{3}$. For Lorenz number, the dot-dashed line denotes the value of the degenerate limit $2.44 \times 10^{-8} \Omega \mathrm{WK}^{-2}$. 
and $b$ are determined by two boundary conditions. The first one is when $E(t)=E_{0}, \omega_{P}$ equals to the value obtained from the traditional formulation, i.e., $\frac{2 N}{g} \sqrt{P} E_{0}$, resulting in $a+b=1$; the second one is $\omega_{P}$ reaches its minimum when $E(t)=0$, and this minimum makes the radius of gyration of ring polymer up to half of the de Broglie wavelength of the corresponding ion. In PIMD simulations, the initial ring polymer is generated according to the Levy-Brown procedure ${ }^{42}$, and the corresponding radius of gyration of ring polymer $R_{g} \propto 1 / \sqrt{T}$. Since $\omega_{P} \propto T$, we have $R_{g}^{2} \propto 1 / \omega_{P}$. Then $a$ and $b$ can be obtained according to the second boundary condition, that is, $b=\left(2 R_{g 0} / \lambda\right)^{2}$, $a$ $=1-\left(2 R_{g 0} / \lambda\right)^{2}$, where $R_{g 0}$ is the radius of gyration of ring polymer when $E(t)=E_{0}, \lambda$ is the de Broglie wavelength of the corresponding ion. In this way, the size of ring polymer is more close to $\lambda$ as the instantaneous kinetic energy of ions is decreased. The importance of this new scheme can be illustrated by considering the dynamics of a hydrogen atom tunnels through a Gaussian potential barrier using the improved centroid PIMD approach. The dependence of the transmission probability on the ratio of the incident energy $E$ to the height of barrier $U_{0}$ is in good agreement with the WKB semi-classical approximation ${ }^{43}$ and the exact results through solving the Schrödinger equation when $\lambda$ is less than the half-height width of the potential barrier (see Supplementary Information). Therefore, this new scheme is more suitable for simulating the nuclear quantum dynamics such as ionic diffusions, since they are dependent on the same physics of scattering or collision.

\section{Electronic structure calculations and MD simulations. All the PIMD and MD} simulations were performed using modified Quantum-ESPRESSO package ${ }^{44}$. A periodic supercell including 250-432 atoms was employed according to different densities, which can ensure convergence of both ionic and electronic properties with good accuracy (see Supplementary Information for convergence tests). The Coulombic pseudopotential ${ }^{45}$ and the generalized-gradient approximation ${ }^{46}$ for exchange-correlation potential were adopted. The kinetic energy cutoff was set from 200 to $400 \mathrm{Ry}$ according to different densities. Electronic distribution was subject to Fermi-Dirac statistics ${ }^{47}$. The Brillouin zone was sampled using the $\Gamma$ point in MD simulations. Denser k-point grids were used to calculate electronic properties.

Within the framework of PIMD, the structural and thermodynamical properties were calculated using the primitive scheme ${ }^{29}$, while the real-time quantum dynamics of nuclei was obtained through the $a b$ initio improved centroid PIMD. The selfdiffusion coefficient was calculated from the slope of the centroid mean square displacement. The shear viscosity was obtained from the autocorrelation function of the off-diagonal components of the stress tensor ${ }^{27}$. Langevin thermostat ${ }^{48}$ was employed to overcome the nonergodic problem, which not only produces a canonical ensemble and compensates the calculated errors ${ }^{45}$, but also gives us an efficient unified description from cold condensed matter to hot dense regime ${ }^{45,49}$. Langevin thermostat was applied only to each noncentroid degree of freedom because thermostats would disturb the dynamical properties of the centroids. The Trotter number was set to 16 after a convergence test. Time step of 2-3 a.u. was used in our ab initio PIMD calculations with 40000 steps after thermalization, while the smaller time steps of $0.2-0.3$ a.u. with $5 \times 10^{5}$ steps and a centroid adiabaticity parameter of 20 were adopted in the centroid PIMD simulations in order to decouple the centroid mode from other normal modes.

The electrical and thermal conductivity and optical absorbtion coefficient were calculated via the Kubo-Greenwood formula ${ }^{50,51}$ with ABINIT package ${ }^{52}$. The electrical conductivity and optical absorption coefficient were calculated with a 0.03 a.u. width of Gaussian smearing and k-point sets of $6 \times 6 \times 6$ and $8 \times 8 \times 8$ for $10 \mathrm{~g} / \mathrm{cm}^{3}$ and $100 \mathrm{~g} / \mathrm{cm}^{3}$, respectively. Here, 10 atomic configurations along the trajectories were employed. In PIMD simulations, the electronic transport properties were obtained by averaging over both PIMD time steps and imaginary time slices.

1. Benoit, M., Marx, D. \& Parrinello, M. Tunnelling and zero-point motion in highpressure ice. Nature 392, 258-261 (1998).

2. Morrone, J. A. \& Car, R. Nuclear quantum effects in water. Phys. Rev. Lett. 101, $017801(2008)$

3. Li, X.-Z., Probert, M. I. J., Alavi, A. \& Michaelides, A. Quantum nature of the proton in water-hydroxyl overlayers on metal surfaces. Phys. Rev. Lett. 104, 066102 (2010).

4. Li, X.-Z., Walker, B. \& Michaelides, A. Quantum nature of the hydrogen bond. Proc. Natl. Acad. Sci. U.S.A. 108, 6369-6373 (2011).

5. Kang, D., Dai, J., Sun, H., Hou, Y. \& Yuan, J. Quantum simulation of thermallydriven phase transition and oxygen K-edge $\mathrm{x}$-ray absorption of high-pressure ice. Sci. Rep. 3, 3272; DOI:10.1038/srep03272 (2013).

6. Biermann, S., Hohl, D. \& Marx, D. Quantum effects in solid hydrogen at ultrahigh pressure. Solid State Commun. 108, 337-341 (1998).

7. Kitamura, H., Tsuneyuki, S., Ogitsu, T. \& Miyake, T. Quantum distribution of protons in solid molecular hydrogen at megabar pressures. Nature 404, 259-262 (2000).

8. Geneste, G., Torrent, M., Bottin, F. \& Loubeyre, P. Strong isotope effect in phase II of dense solid hydrogen and deuterium. Phys. Rev. Lett. 109, 155303 (2012).

9. Morales, M. A., McMahon, J. M., Pierleoni, C. \& Ceperley, D. M. Nuclear quantum effects and nonlocal exchange-correlation functionals applied to liquid hydrogen at high pressure. Phys. Rev. Lett. 110, 065702 (2013).

10. Chen, J. et al. Quantum simulation of low-temperature metallic liquid hydrogen. Nat. Commun. 4, 2064 (2013).
11. Yonetani, Y. \& Kinugawa, K. Centroid molecular dynamics approach to the transport properties of liquid para-hydrogen over the wide temperature range. J. Chem. Phys. 120, 10624-10633 (2004).

12. Hunter, G. \& Kuriyan, M. Proton collisions with hydrogen atoms at low energies: quantum theory and integrated cross-sections. Proc. R. Soc. London A 353, 575-588 (1977).

13. Fortov, V. E. Extreme States of Matter (Springer, Berlin, Heidelberg, 2011).

14. McMahon, J. M., Morales, M. A., Pierleoni, C. \& Ceperley, D. M. The properties of hydrogen and helium under extreme conditions. Rev. Mod. Phys. 84, 1607-1653 (2012).

15. Lambert, F., Recoules, V., Decoster, A., Clérouin, J. \& Desjarlais, M. On the transport coefficients of hydrogen in the inertial confinement fusion regime. Phys. Plasmas 18, 056306 (2011).

16. Guillot, T. Interiors of giant planets inside and outside the solar system. Science 286, 72-77 (1999).

17. Lissauer, J. J. Extrasolar planets. Nature 419, 355-358 (2002).

18. Daligault, J. Diffusion in ionic mixtures across coupling regimes. Phys. Rev. Lett. 108, 225004 (2012).

19. Fortney, J. J. \& Nettelmann, N. The interior structure, composition, and evolution of giant planets. Space Sci. Rev. 152, 423-447 (2010).

20. Militzer, B. Equation of state calculations of hydrogen-helium mixtures in solar and extrasolar giant planets. Phys. Rev. B 87, 014202 (2013).

21. Swift, D. C. et al. Mass-radius relationships for exoplanets. Astrophys. J. 744, 59 (2012).

22. Pozzo, M., Davies, C., Gubbins, D. \& Alfè, D. Thermal and electrical conductivity of iron at Earth's core conditions. Nature 485, 355-358 (2012).

23. Xu, B. \& Hu, S. X. Effects of electron-ion temperature equilibration on inertial confinement fusion implosions. Phys. Rev. E 84, 016408 (2011).

24. Loubeyre, P. et al. Coupling static and dynamic compressions: first measurements in dense hydrogen. High Press. Res. 24, 25-31 (2004).

25. Jeanloz, R. et al. Achieving high-density states through shock-wave loading of precompressed samples. Proc. Natl. Acad. Sci. U.S.A. 104, 9172-9177 (2007).

26. Collins, L. A. et al. Dynamical and optical properties of warm dense hydrogen. Phys. Rev. B 63, 184110 (2001).

27. Alfe, D. \& Gillan, M. J. First-principles calculation of transport coefficients. Phys. Rev. Lett. 81, 5161-5164 (1998).

28. Holst, B., French, M. \& Redmer, R. Electronic transport coefficients from ab initio simulations and application to dense liquid hydrogen. Phys. Rev. B 83, 235120 (2011).

29. Marx, D. \& Parrinello, M. Ab initio path integral molecular dynamics: Basic ideas. J. Chem. Phys. 104, 4077-4082 (1996).

30. Tuckerman, M. E., Marx, D., Klein, M. L. \& Parrinello, M. Efficient and general algorithms for path integral Car-Parrinello molecular dynamics. J. Chem. Phys. 104, 5579-5588 (1996).

31. Marx, D., Tuckerman, M. E. \& Martyna, G. J. Quantum dynamics via adiabatic $a b$ initio centroid molecular dynamics. Comput. Phys. Commun. 118, 166-184 (1999).

32. Krilov, G. \& Berne, B. J. Real tim quantum correlation functions. I. Centroid molecular dynamics of anharmonic systems. J. Chem. Phys. 111, 9140-9146 (1999).

33. Polyakov, E. A., Lyubartsev, A. P. \& Vorontsov-Velyaminov, P. N. Centroid molecular dynamics: Comparison with exact results for model systems. J. Chem. Phys. 133, 194103 (2010).

34. Edwards, M. J. et al. The experimental plan for cryogenic layered target implosions on the National Ignition Facility-The inertial confinement approach to fusion. Phys. Plasmas 18, 051003 (2011).

35. Bermejo, F. J. et al. Quantum effects on liquid dynamics as evidenced by the presence of well-defined collective excitations in liquid para-hydrogen. Phys. Rev. Lett. 84, 5359-5362 (2000).

36. Liberatore, E., Pierleoni, C. \& Ceperley, D. M. Liquid-solid transition in fully ionized hydrogen at ultra-high pressures. J. Chem. Phys. 134, 184505 (2011).

37. Belonoshko, A. B., Ramzan, M., Mao, H.-K. \& Ahuja, R. Atomic diffusion in solid molecular hydrogen. Sci. Rep. 3, 2340; DOI:10.1038/srep02340 (2013).

38. Hansen, J.-P. \& McDonald, I. R. Theory of Simple Liquids (Academic Press, 2011).

39. Dai, J. et al. Structure, equation of state, diffusion and viscosity of warm dense Fe under the conditions of a giant planet core. New J. Phys. 15, 045003 (2013).

40. Recoules, V., Lambert, F., Decoster, A., Canaud, B. \& Clérouin, J. Ab initio determination of thermal conductivity of dense hydrogen plasmas. Phys. Rev. Lett. 102, 075002 (2009).

41. Pozzo, M., Desjarlais, M. P. \& Alfe, D. Electrical and thermal conductivity of liquid sodium from first-principles calculations. Phys. Rev. B 84, 054203 (2011)

42. Fosdick, L. D. \& Jordan, H. F. Path-integral calculation of the two-particle Slater sum for $\mathrm{He}^{4+}$. Phys. Rev. 143, 58-66 (1966).

43. Razavy, M. Quantum Theory of Tunneling (World Scientific, Singapore, 2003).

44. Giannozzi, P. et al. QUANTUM ESPRESSO: a modular and open-source software project for quantum simulations of materials. J. Phys.: Condens. Matter 21, 395502 (2009).

45. Dai, J., Hou, Y. \& Yuan, J. Unified first principles description from warm dense matter to ideal ionized gas plasma: Electron-ion collisions induced friction. Phys. Rev. Lett. 104, 245001 (2010)

46. Perdew, J. P., Burke, K. \& Ernzerhof, M. Generalized gradient approximation made simple. Phys. Rev. Lett. 77, 3865-3868 (1996). 
47. Mermin, N. D. Thermal properties of the inhomogeneous electron gas. Phys. Rev. 137, A1441-A1443 (1965).

48. Gillan, M. J. The quantum simulation of hydrogen in metals. Phil. Mag. A 58, 257-283 (1988).

49. Dai, J., Kang, D., Zhao, Z., Wu, Y. \& Yuan, J. Dynamic ionic clusters with flowing electron bubbles from warm to hot dense iron along the Hugoniot curve. Phys. Rev. Lett. 109, 175701 (2012).

50. Kubo, R. Statistical-mechanical theory of irreversible processes. I. General theory and simple applications to magnetic and conduction problems. J. Phys. Soc. Jpn. 12, 570-586 (1957).

51. Greenwood, D. A. The Boltzmann equation in the theory of electrical conduction in metals. Proc. Phys. Soc. London 71, 585-596 (1958).

52. Gonze, X. et al. ABINIT: First-principles approach to material and nanosystem properties. Comput. Phys. Commun. 180, 2582-2615 (2009).

\section{Acknowledgments}

This work is supported by the National Basic Research Program of China (973 Program) under Grant No. 2013CB922203, the National Natural Science Foundation of China under Grant Nos. 11104351, 11274383. Calculations were carried out at the Research Center of Supercomputing Application, NUDT.

\section{Author contributions}

D.K., J.D. and J.Y. designed the research. D.K. and J.D. modified the code. D.K., H.S. and W.C. performed all calculations. D.K., H.S., J.D., Z.Z., Y.H. and J.Z. analysed the data. D.K., J.D., Z.Z. and J.Y. wrote the paper. All authors reviewed the manuscript.

\section{Additional information}

Supplementary information accompanies this paper at http://www.nature.com/ scientificreports

Competing financial interests: The authors declare no competing financial interests. How to cite this article: Kang, D. et al. Nuclear quantum dynamics in dense hydrogen. Sci. Rep. 4, 5484; DOI:10.1038/srep05484 (2014).

This work is licensed under a Creative Commons Attribution-NonCommercialNoDerivs 4.0 International License. The images or other third party material in this article are included in the article's Creative Commons license, unless indicated otherwise in the credit line; if the material is not included under the Creative Commons license, users will need to obtain permission from the license holder in order to reproduce the material. To view a copy of this license, visit http:// creativecommons.org/licenses/by-nc-nd/4.0/ 